\title{
Tornare alla contemporaneità. Introduzione al dossier sui Cantieri della didattica
}

\author{
di Carla Marcellini e Andrea F. Saba
}

Mar 31, 2020 | In evidenza, Insegnare la contemporaneità oggi | $\underline{0}$ |
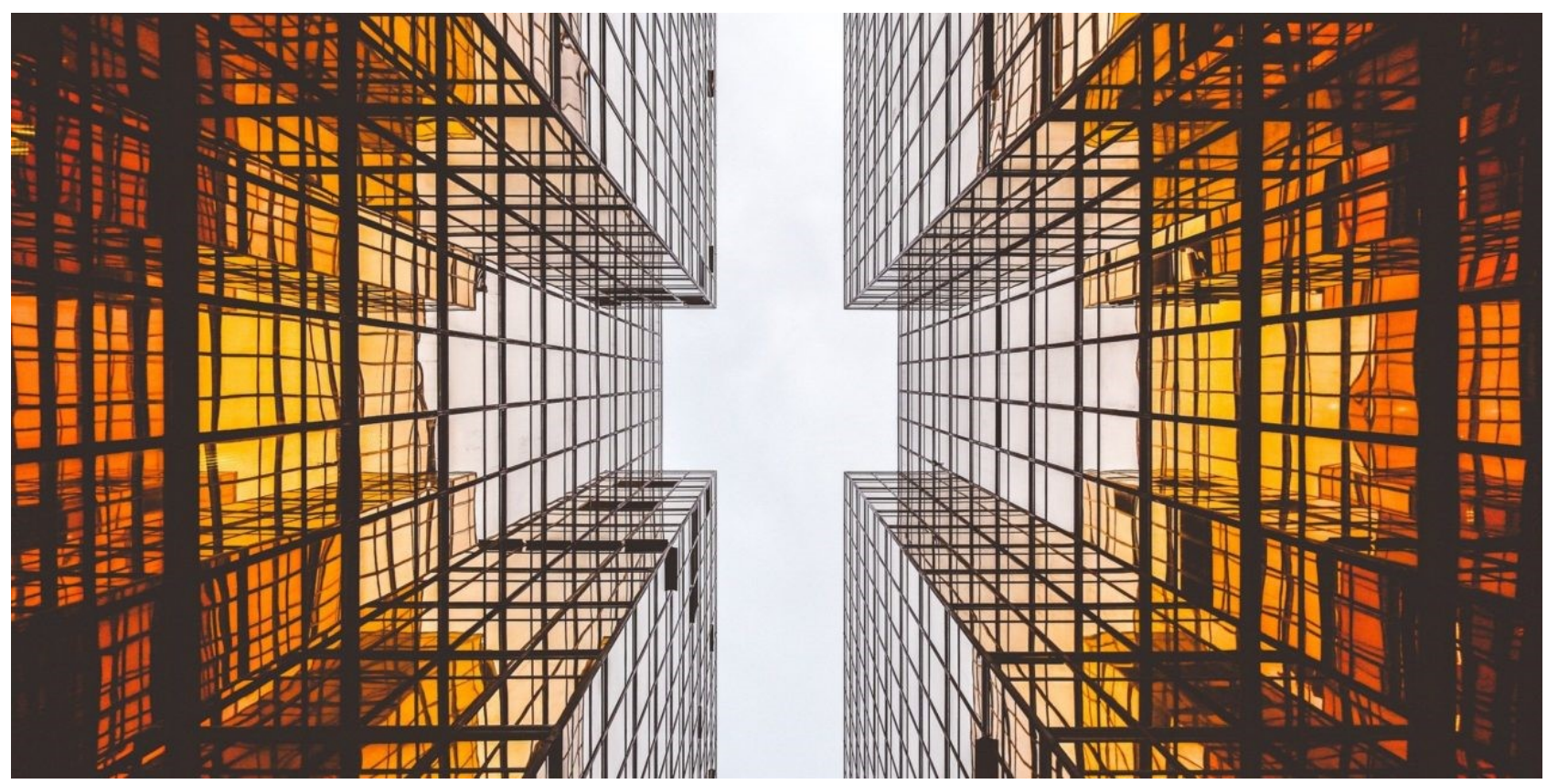

\section{Abstract}

A maggio 2019 si sono svolti a Rimini i Cantieri della Didattica Insegnare la contemporaneità, 1948-2018, promossi dall'Istituto nazionale Ferruccio Parri, con l'obiettivo di riflettere su come si insegna nella scuola italiana la storia della Seconda metà del Novecento. In questo Dossier si darà conto dei problemi affrontati, delle relazioni, dei laboratori e delle esperienze presentate.

\section{I problemi strutturali}

Se è evidente che non ci sarebbero ostacoli di sorta al fatto che preparazione disciplinare, metodologia didattica e creatività consentirebbero agli insegnanti di storia di dare risposte rapide ed efficaci nella costruzione di curricoli - modelli, contenuti e strategie - e di validi percorsi per l'insegnamento della storia contemporanea, tuttavia nella pratica quotidiana questo virtuoso processo non è affatto scontato.

In primo luogo resta forte l'abitudine, cristallizzata nelle Indicazioni nazional (e solo in parte rivoluzionata nelle riforme del 2017 per gli Istituti professionali), di dare rilevanza cronologica e sequenziale degli eventi storici. Nel quadro orario ridotto, conseguenza delle riforme di ormai tre lustri (compresa l'Alternanza scuola-lavoro, ora ridimensionata nei Percorsi per le competenze trasversali e l'orientamento), la scansione degli argomenti rimane in ordine cronologico con scarse aperture a trattare la storia per nuclei problematici o tematici. Un'apertura in questo senso permetterebbe ad esempio di poter affrontare più distesamente la sezione contemporaneistica nella quinta classe della secondaria di secondo grado, ritornando su o ripartendo da quei nuclei.[1] Nella 
scuola primaria è possibile lavorare sulla contemporaneità anche se la scansione temporale della programmazione non la prevede, nella secondaria di primo grado invece la storia contemporanea è inserita nel curricolo dell'ultimo anno, anch'essa scandita cronologicamente.[2]

Gli altri nodi irrisolti di una disciplina come la storia, che tratta la complessità e che lotta nelle aule scolastiche per accendere l'interesse degli studenti, sono costituiti dal sostanziale vuoto didattico non contenutistico - relativo agli ultimi trent'anni. Si tratta di una situazione niente affatto semplice, generata da diversi fattori: dal mancato adeguamento delle Indicazioni che si fermano, sostanzialmente, alla caduta del muro di Berlino e per l'Italia alla crisi istituzionale del 1992; dalla preparazione storica spesso non specifica dei docenti a cui è affidato l'insegnamento della storia (in Italia il docente di storia insegna sempre anche un'altra materia, e raramente ha una laurea in storia); dalle carenze rilevate in strumenti come i manuali che, nonostante le poderose dimensioni, solo raramente propongono tematizzazioni efficaci o periodizzazioni significative per la storia recente.[3] I testi scolastici si limitano spesso a citare i fatti del nostro mondo contemporaneo, elencandoli in una piatta sequenza cronachistica, priva dello spessore che ne favorirebbe la comprensione. Anche gli strumenti digitalizzati a corredo del testo principale mostrano molti limiti che andrebbero indagati e valutati in maniera approfondita.[4]

In realtà proposte per dare forma a un manuale differente non mancano. "Novecento.org", ad esempio, ha più volte dedicato la propria attenzione a identificare strumenti - si pensi allo studio di caso - che potrebbero aiutare lo studente a guardare alla disciplina con un approccio diverso, più coinvolgente oltre che più efficace nello sviluppo di competenze disciplinari e trasversali.[5]

\section{Dalla storiografia alla pratica didattica}

Si può sempre più spesso notare come la percezione comune del passato possa risultare viziata da incursioni contingenti della politica. Sulla storia resistenziale e sull'immediato dopoguerra, ad esempio è accaduto e accade non di rado. Una risposta più consolidata e condivisa riguarda il dibattito storiografico sulla fine dell'Italia liberale e poi sull'inizio del periodo repubblicano, mentre restano vivaci i toni sulle crisi di lungo periodo, sia quella politico-istituzionale, che ha travolto i partiti tradizionali nel 1992-1993, sia quella su cui si è innestato il declino economico dell'Italia.[6] 


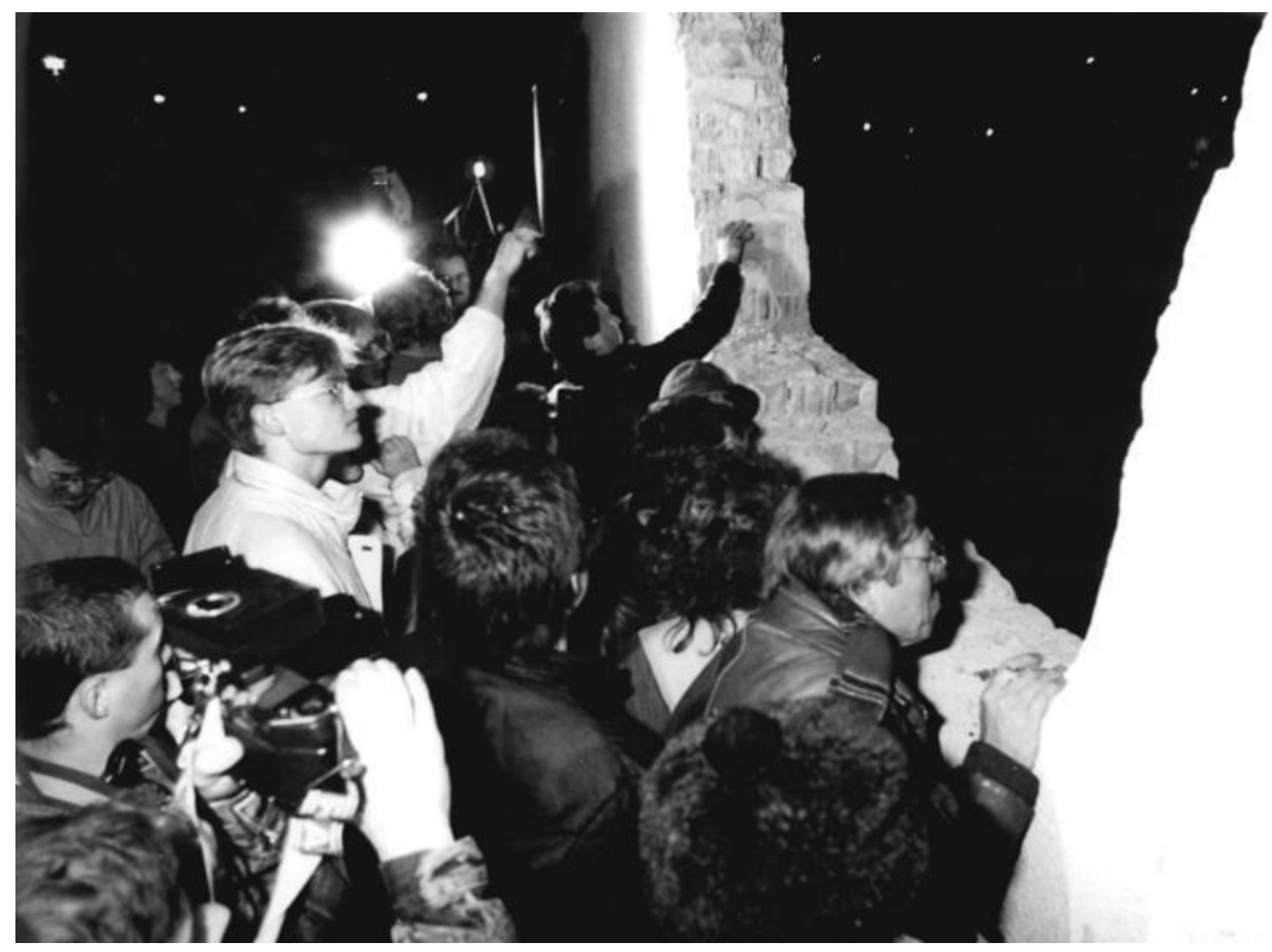

Con una breccia nel Muro si riapre la frontiera sulla Eberswalder Strasse a Berlino, 11 novembre 1989 (R. Roeske, Bundesarchiv 183-1989-1111-005, CC-BY-SA 3.0)

Insegnare la dimensione di complessità propria dell'età contemporanea comporta non solo prendere in considerazione le analisi storiografiche in modalità più tradizionale, ma anche le forme di arte $\mathrm{e}$ di rappresentazione più disparate, che colgono gli eventi storici del presente e che probabilmente continueranno a raccontarli anche in un futuro più lontano, consentendone la cristallizzazione nella memoria e nella storiografia. Fotografie, graphic novel, serial, docufilm e film, rappresentazioni teatrali, storie di persone comuni, testimonianze, percezioni, addirittura giochi di ruolo o giochi virtuali ci aprono uno spiraglio e ci guidano sui fatti epocali e possono intercettare i linguaggi con cui i giovani oggi acquisiscono le loro conoscenze e sviluppano le loro competenze. Grazie alla disponibilità di strumenti di relativa semplicità d'uso, gli studenti spesso trasformano le competenze e le conoscenze acquisite in "prodotti" scolastici come video e app, favorendone circolazione e visibilità, oltre che fra i docenti e gli studenti, nell'ambito delle comunità educative e alle istituzioni scolastiche.[8]

\section{Verso l'histotainment?}

Nel settore dell'informazione abbiamo assistito, alla semplificazione dei contenuti, con le notizie e la cronaca che dai quotidiani cartacei sono divenute dominio della televisione, che per la loro comunicazione usa forme di intrattenimento come talk show e servizi di durata rapidissima, corredati da grafiche accattivanti (con una commistione verso lo spettacolo che ha dato luogo all'infotainment).[9] L'irrompere di forme di trasmissione meno tradizionali del testo scritto, ha parallelamente dato luogo in campo educativo - soprattutto accademico - all'offerta di formazione 
con modalità legate a particolari eventi (si pensi alle lezioni di Ted Talks) o a interi corsi. Anche in campo storico, così come per altre discipline, rileviamo che si va verso la semplificazione di contenuti disciplinari, per ottenere una maggiore diffusione e coinvolgimento del pubblico potenziale, con la costruzione perciò di una specifica branca che utilizza forme di intrattenimento e che possiamo battezzare qui come histotainment: nuove modalità di comunicazione che possiamo tuttavia far afferire più alla public history che alla didattica della storia.[10]

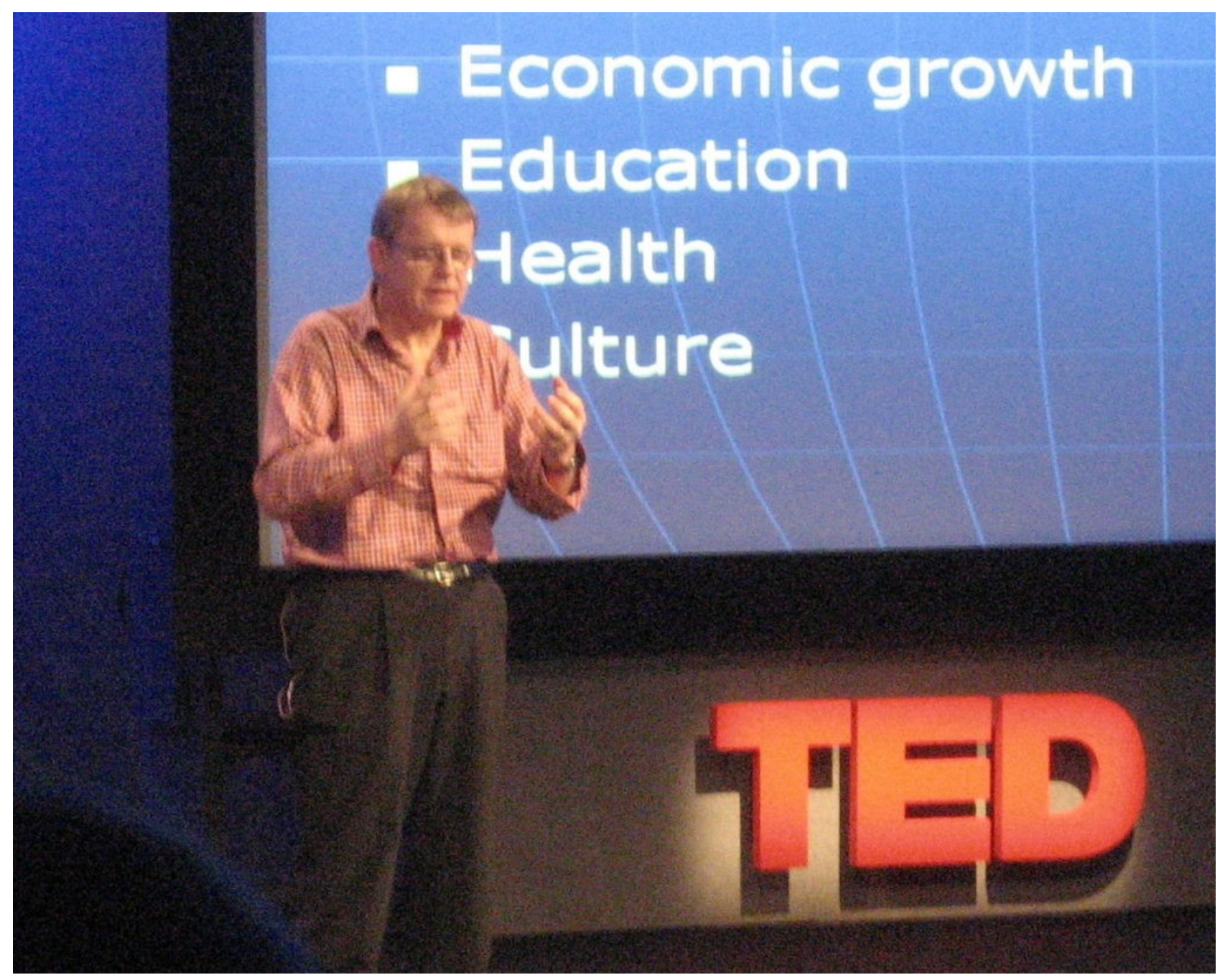

Un classico dell'edutainment: il Ted Talk di H. Rosling, medico specialista di salute pubblica e membro della Reale accademia delle scienze di Svezia, 7 marzo 2007

D'altronde, l'adattamento alle forme contemporanee di trasmissione del sapere è un'operazione necessaria nella società della contemporaneità, caratterizzata dalla crescente velocità della comunicazione a scapito della profondità strutturale delle informazioni veicolate: «oggi manca il tempo, noi amiamo conoscere rapidamente, attraverso una conferenza, un dibattito, o la lettura non di ponderosi volumi, ma di brevi saggi che ci dicano l'essenziale [...] senza prolisse analisi e ragionamenti complessi e involuti», osservavano i giovani del 1971 a un «perplesso» Pietro Secchia, che si accomodò, tuttavia, a produrre un agile libretto sul fascismo, in funzione dell'attualità che allora lo riportava al centro della violenza politica.[11] 
Con una lunga esperienza di formazione didattica, la rete nazionale Parri ha risposto e risponde costantemente alle sollecitazioni che vengono, ancor più che dalle scuole, dalla necessità intellettuale e professionale degli insegnanti di capire la storia contemporanea e di sviluppare strumenti e metodologie che ne favoriscano l'apprendimento. Con questo sguardo, in occasione dei Cantieri della didattica di Rimini, è stata realizzata una ricerca interna per capire quali e quanti prodotti didattici e pacchetti di formazione siano stati realizzati dalla rete degli istituti rispetto al Secondo Novecento. Le esperienze più significative sono state presentate nel corso del seminario, mentre un quadro generale ci ha permesso di fare un bilancio, seppur provvisorio, da cui emerge una grande ricchezza tematica, ma con un prevalere di lavori di storia nazionale, lasciando scoperti ampi spazi del mondo (es la storia dell'America latina, dell'Asia e dell'Africa sub-sahariana).[12]

Dal punto di vista tematico le buone pratiche presentate spaziano dalla questione della democrazia e della Costituzione, all'emigrazione interna, al consumismo, al femminismo e alla violenza politica, al Sessantotto, fino allo smantellamento industriale postfordista di Sesto San Giovanni. [13]

Le modalità hanno pure spaziato. Si va dai laboratori con l'uso di canzoni italiane del periodo 19581982 usate a margine e a integrazione della mostra Noi... non erano solo canzonette, allestita a Torino e Bologna, a quelli che prevedono la musica della protesta giovanile statunitense resa disponibile grazie allo sviluppo di mappe geostoriche su Google StoryMap[14], a lezioni e laboratori con documenti archivistici più tradizionali che si accompagnano alla visita di aree di archeologia industriale informale e della città di Sesto San Giovanni e dalla lettura del territorio nelle sue trasformazioni secolari; fino alla web serie in forma di dialoghi, registrati fra studenti adulti e testimoni, divenuti l'occasione per la discussione del tempo presente come punto d'arrivo dell'evoluzione storica e dell'applicazione dei principi costituzionali.

Note:

[1] Cfr. Dm 211/2010 “Indicazioni Nazionali” e il Dlgs 61/2017e le collegate Linee guida per favorire e sostenere l'adozione del nuovo assetto didattico e organizzativo dei percorsi di istruzione professionale, Miur, s.d. in https://www.miur.gov.it/web/guest/istituti-professionali, url consultata il 23-02-2020.

[2] Cfr. in questo dossier G. Gabrielli, La scuola primaria a confronto con la contemporaneità, in "Nocecento.org", 13, 2020.

[3] Cfr. in questo dossier, anche per qualche impostazione innovativa, C. Marcellini e A. Portincasa, I manuali scolastici di storia contemporanea: una panoramica, in "Novecento.org", 13, 2020.

[4] Il tema meriterebbe un approfondimento e una ricerca che riesca a valutare l'ampia gamma di materiali presenti nelle espansioni digitali dei manuali. Ci si limita qui a segnalare i contributi del 2015: M. Flores, I testi manualistici dell'odierna editoria scolastica e le loro integrazioni digitali. Un'analisi dell'offerta digitale dell'editoria scolastica-Parte 1, L. Cajani, Le estensioni on-line dei manuali italiani di storia contemporanea: elementi per un'analisi didattica. Un'analisi dell'offerta digitale dell'editoria scolastica-Parte 2, e M.B. Ligorio, Modelli pedagogici, potenzialità e limiti dei prodotti multimediali a supporto dei testi didattici. Un'analisi dell'offerta digitale dell'editoria scolastica-Parte 3, tutti in "Novecento.org", 5, dicembre 2015. Per un dibattito in cui le Indicazioni nazionali costituiscono un punto problematico cfr. A. Portincasa, Cinque domande sulla didattica 
della storia. Intervista a Salvo Adorno, Agostino Bistarelli, Gianluca Cuniberti, Vito Lorè, Walter Panciera, in "Novecento.org", 12, 2019.

[5] Cfr. A. Brusa (a cura di), Più di un manuale. Gli strumenti per insegnare la storia della Repubblica, in "Novecento.org", 8, 2017 e Portincasa, Cinque domande cit.

[6] Cfr. in questo dossier U. Berti Arnoaldi, Che cos'è oggi la contemporaneità, in "Novecento.org", 13, 2020.

[7] Cfr. in questo dossier - anche per la citazione - F. Cammarano, La Storia contemporanea. Una prospettiva, in "Novecento.org", 13, 2020. Si veda anche R. Bodei, Se la storia ha un senso, Moretti \& Vitali, Bergamo, 2006.

[8] Cfr. in questo dossier C. Greppi, In fuga dal presente. Fonti e linguaggi per narrare la contemporaneità, in "Novecento.org", 13, 2020: la citazione è in Ibid.

[9] Sull'infotainment, definizione di fenomeni quanto mai disparati che vanno dalle soft news a trasmissioni televisive di informazione e dibattito, cfr. per esempio, per il caso italiano, R. Stella, Box populi. Il sapere e il fare della neotelevisione, Donzelli, Roma 1999.

[10] In generale, per la didattica si parla di edutainment, termine coniato da Walt Disney: sulla sua opera cfr. A. Bowdoin Van Riper (a cura di), Learning from Mickey, Donald and Walt. Essays on Disney's Edutainment Films, McFarland and Co., Jefferson (NC), 2011. Il campo è vasto e abbastanza studiato: per alcune definizioni cfr. K.Rapeepisarn, K.Wong, L.Fung e A.Depickere, Similarities and Differences between "learn through play" and "edutainment", in K.K.W. Wong, L.C.C. Fung, P. Cole e Y. Pisan (a cura di), Proceedings of the 3rd Australasian Conference on Interactive Entertainment, 4-6 December 2006, Murdoch University, Murdoch (W. Australia) 2006, pp. 28-32, F. Cervellini e D. Rossi, Comunicare emozionando. L'edutainment per la comunicazione intorno al patrimonio culturale, in "Disegnarecon", 8, 2011, pp. 49-55 e il dibattito in A. Giglio e I. Messina, L'edutainment nella didattica delle lingue: nemico o alleato?, in “Lingu@ggi 21.0”, 1,2010, https://www.researchgate.net/publication/259658895_L'edutainment_nella_didattica_delle_lingue nemico_o_alleato, url consultata il 15 febbraio 2020. Non va, peraltro, sottovalutato 1'aspetto economico connesso a questi sviluppi: cfr. G. Beato, Turning to Education for Fun, in "New York Times", 19 marzo 2015.

[11] P. Secchia, Le armi del fascismo 1921-1971, Feltrinelli, Milano 1971, p. 7.

[12] Cfr. in questo dossier F. Febbraro e A.F. Saba, Gli Istituti della Rete Parri e la didattica sul periodo "dopo la Costituzione", in "Novecento.org", 13, 2020.

[13] Cfr. in questo dossier P. Mencarelli, La Costituzione è giovane. Web serie in sei episodi, A.G. Salassa, Fare storia del secondo Novecento con le canzoni italiane. 5 laboratori proposti da Istoreto, F.M. Ferrara, Secondo Novecento e didattica digitale: "Il Sessantotto" e "Another brick in the Wall”, M. Di Barbora, Insegnare la storia del lavoro. L'esperienza di Fondazione Isec.

[14] Sulla piattaforma digitale didattica E-Story cfr. A. Portincasa e I. Pizzirusso, Il digitale come risorsa per la didattica laboratoriale, in "Novecento.org", 11, 2019. 